\title{
AN INTEGRALLY CLOSED RING WHICH IS NOT THE INTERSECTION OF VALUATION RINGS
}

\author{
JOACHIM GRÄTER
}

(Communicated by Louis J. Ratliff, Jr.)

\begin{abstract}
Each commutative ring $R$ which is integrally closed in its total quotient ring $T(R)$ is the intersection of all paravaluation rings of $T(R)$ containing $R$. In this note an example is given that shows that this statement is not true with "valuation rings" instead of "paravaluation rings". This is an answer of a question asked by J. A. Huckaba in [3].
\end{abstract}

In this note, all rings are commutative. Every ring has a unit-element, denoted by 1 , which is preserved by homomorphisms and inherited by subrings.

Let $\Gamma$ be a totally ordered commutative group written additively and let $\Gamma_{\infty}=\Gamma_{U}\{\infty\}$ where $\gamma<\infty, \gamma+\infty=\infty+\gamma=\infty+\infty=\infty$ is defined for all $\gamma \in \Gamma$. If $R$ is a ring then a mapping

$$
v: R \rightarrow \Gamma_{\infty}, \quad r \mapsto v(r),
$$

is called a paravaluation if the following hold for all $x, y \in R$ :

(a) $v(x y)=v(x)+v(y)$.

(b) $v(x+y) \geq \min \{v(x), v(y)\}$.

(c) $v(1)=0$ and $v(0)=\infty$.

Furthermore, a surjective paravaluation is called a valuation and a subring $B$ of $R$ is called a valuation ring, resp. a paravaluation ring, if there is a valuation, resp. paravaluation, $v$ of $R$ such that $B=\{r \in R \mid v(r) \geq 0\}$. In this case, we write $B=B_{v}$ and $M_{v}:=\{r \in R \mid v(r)>0\}$ is a prime ideal of $B_{v}$.

Paravaluation rings are a useful tool in commutative ring theory for describing the integral closure of a ring:

Theorem. Let $R$ be a ring with total quotient ring $T$. Then $R$ is integrally closed (in $T$ ) if and only if $R$ is the intersection of its set of paravaluation rings of $T$ containing $R$.

This statement is also valid if $T$ is an arbitrary ring and $R$ a subring of $T$ (cf. [2] and [3, Theorem 9.1]). In his new book, J. A. Huckaba asked whether

Received by the editors September 27, 1988, and in revised form, November 17, 1988.

1980 Mathematics Subject Classification (1985 Revision). Primary 13B20; Secondary 13A18.

Key words and phrases. Commutative rings, integral closure, valuation rings. 
or not "paravaluation" can be replaced by "valuation" in the statement of the Theorem (cf. [3, p. 54 and p. 82]). In this note, we give an example which shows that "paravaluation" cannot be so replaced.

Let $K$ be a commutative field and let $R=K[t]$ be the polynomial ring in the indeterminate $t$ over $K$.

Proposition 1. For each $f \in R \backslash K$ there exists a ring $S_{f}$ such that the following hold:

(i) $R$ is a subring of $S_{f}$.

(ii) There exists a nonzero $z_{f} \in S_{f}$ such that $f \cdot z_{f}=0$ and $z_{f} \cdot z_{f}=0$.

(iii) $R \cap R z_{f}=\{0\}$.

Proof. Let $x$ be an indeterminate over $R$ and let $J$ be the ideal of $R[x]$ generated by $x f$ and $x^{2}$. We define $S:=R[x] / J$.

(i) $\Phi: R \rightarrow S, r \mapsto r+J$. $\Phi$ is a monomorphism.

(ii) $z_{f}:=x+J$.

(iii) Let $g, h \in R$ such that $g=h z_{f}$. We obtain $g-h x \in x f R[x]+x^{2} R[x]$ and $g \in x R[x]$ follows.

Proposition 2. There exists a ring $S$ such that the following hold:

(i) $R$ is a subring of $S$.

(ii) For each $f \in R \backslash K$ there exists $s_{f} \in S, s_{f} \neq 0$, such that $f \cdot s_{f}=0$ and $s_{f} \cdot s_{f}=0$.

(iii) $s_{f} \cdot s_{g}=0$ holds for all $f, g \in R \backslash K$.

(iv) $R\left[s_{f} \mid f \in R \backslash K\right]=R \oplus\left(\bigoplus_{f \in R \backslash K} R \cdot s_{f}\right)$.

Proof. Let $I=R \backslash K$ be an index set. For each $f \in I$ let $S_{f}$ be a ring satisfying (i), (ii), (iii) of Proposition 1 and let $S:=\prod_{f \in I} S_{f}$ be the direct product of $\left\{S_{f} \mid f \in I\right\}$.

Since $\Phi: R \rightarrow S, r \mapsto(r)$ is a monomorphism, (i) is valid. Furthermore, we define for each $f \in R \backslash K$

$$
s_{f}: I \rightarrow \bigcup_{i \in I} S_{i}, \quad s_{f}(i)= \begin{cases}z_{f} & i=f, \\ & \text { if } \quad \\ 0 & i \neq f,\end{cases}
$$

and (ii), (iii), (iv) are obvious.

Now, let $S$ be a ring satisfying (i)-(iv) of Proposition 2. We define

$$
A:=R\left[s_{f} \mid f \in R \backslash K\right] .
$$

If $P$ denotes the ideal of $A$ generated by all $s_{f}, f \in R \backslash K$, we get that $P$ is the nilradical of $A$ and $P$ is prime by $A / P \cong R$. Furthermore, each $a \in A$ can be written in one and only one way in the form

$$
a=f+p
$$


where $f \in R$ and $p \in P$. By construction, we obtain

$a\left(=f+p\right.$ with $f \in R, p \in P$ ) is regular in $A$ if and only if $f \in K^{\times}$.

If $a$ is not regular then $a \cdot p=0$ holds for a suitable nonzero $p \in P$.

Now, let $v: K \rightarrow \Gamma_{\infty}$ be a valuation of $K$ of height 1, i.e. $B_{v}$ is the only proper subring of $K$ containing $B_{v}$. We define

$$
B:=\left\{b_{0}+b_{1} t+\cdots+b_{n} t^{n} \mid n \in \mathbf{N}, b_{0} \in B_{v}, b_{1}, \ldots, b_{n} \in M_{v}\right\}
$$

and

$$
V:=\{f+p \mid f \in B, p \in P\} .
$$

$B$ is a subring of $R$ and $V$ is a subring of $A$. By $P \subseteq V$ and $(*)$, each $f+p \in V$ is regular in $V$ if and only if $f \in B_{v}, f \neq 0$. If $Q(V)$ denotes the total quotient ring of $V$ then

$$
\begin{array}{ccc}
\pi: Q(V) & \rightarrow & K[t] \\
(f+p) \cdot(b+q)^{-1} & \mapsto & f \cdot b^{-1}
\end{array}
$$

is an epimorphism with $\operatorname{ker} \pi=P Q(V)$ where $f \in B, b \in B_{v}, b \neq 0$ and $p, q \in P$. Since $P$ is nilpotent, $u(P)=\{\infty\}$ holds for each paravaluation of $Q(V)$. Therefore, $\pi$ induces a bijection between the set of all valuation rings, resp. paravaluation rings, of $Q(V)$ and the set of all valuation rings, resp. paravaluation rings, of $K[t]$. By $\pi(V)=B$, we are done if we know that $B$ is a paravaluation ring of $K[t]$ which is not equal to the intersection of all valuation rings of $K[t]$ containing $B$.

(A) $B$ is a paravaluation ring of $K[t]$. We define a valuation $w$ of $K(t)$ such that $B_{w} \cap K[t]=B$. There exists a totally ordered commutative group $\Gamma^{\prime}$ written additively and an element $\xi$ of $\Gamma^{\prime}$ such that $\Gamma$ is a subgroup of $\Gamma^{\prime}$ and $0<n \xi<\gamma$ holds for all $\gamma \in \Gamma, \gamma>0$, and $n \in \mathbf{N}$. By [1, Chap. VI, $\S 10.1$ Prop. 1], there exists a unique extension $w$ of $v$ to $K(t)$ with values in $\Gamma_{\infty}^{\prime}$ such that $w(t)=-\xi$. Let $f=k_{0}+k_{1} t+\cdots+k_{n} t^{n}$ and $w(f)=\min \left\{v\left(k_{0}\right)\right.$, $\left.v\left(k_{1}\right)-\xi, \cdots, v\left(k_{n}\right)-n \xi\right\}$. We obtain

$$
\begin{aligned}
w(f) \geq 0 & \Leftrightarrow v\left(k_{0}\right), v\left(k_{1}\right)-\xi, \cdots, v\left(k_{n}\right)-n \xi \geq 0 \\
& \Leftrightarrow v\left(k_{0}\right) \geq 0, v\left(k_{1}\right) \geq \xi, \cdots, v\left(k_{n}\right) \geq n \xi \\
& \Leftrightarrow v\left(k_{0}\right) \geq 0, v\left(k_{1}\right)>0, \cdots, v\left(k_{n}\right)>0 \\
& \Leftrightarrow f \in B .
\end{aligned}
$$

(B) $B_{v}[t]$ is the intersection of all valuation rings of $K[t]$ containing $B$. First of all, $B_{v}[t]$ is a valuation ring of $K[t]$ since

$$
\begin{array}{ccc}
u: K[t] & \longrightarrow & \Gamma_{\infty} \\
k_{0}+\cdots+k_{n} t^{n} & \rightarrow & \min \left\{v\left(k_{0}\right), \cdots, v\left(k_{n}\right)\right\}
\end{array}
$$

is a valuation of $K[t]$ such that $B_{u}=B_{v}[t]$.

Now, let $u$ be an arbitrary valuation of $K[t]$ such that $B \cong B_{u}$. If $K \cong B_{u}$ then $B_{u}=K[t]$ follows by $B \leqq B_{u}$. Thus, let $K \nsubseteq B_{u}$. Since $v$ has height 
1, we obtain $B_{u} \cap K=B_{v}$. Clearly, $u(t) \geq 0$ implies $B_{v}[t] \subseteq B_{u}$. Thus, let $u(t)<0$. Then, $u\left(t^{n}\right)<u(k)<0$ cannot hold for any $k \in K, n \in \mathbf{N}$. Otherwise we obtain $u\left(k^{-1} t^{n}\right)<0$ for at least one $k^{-1} \in M_{v}$ and one $n \in \mathbf{N}$, i.e. $k^{-1} t^{n} \notin B_{u}$ but $k^{-1} t^{n} \in B$. Therefore, $u(k)<u\left(t^{n}\right)<0$ is valid for all $k \in K \backslash B_{v}$ and $n \in \mathbf{N}$. Thus, $n u(t) \notin \Gamma$ holds for all $n \in \mathbf{N}$. By [1, Chap. VI, §10.1 Prop.1],

$$
u\left(k_{0}+k_{1} t+\cdots+k_{n} t^{n}\right)=\min \left\{v\left(k_{0}\right), v\left(k_{1}\right)+u(t), \cdots, v\left(k_{n}\right)+n u(t)\right\}
$$

follows for all $k_{0}, k_{1}, \cdots, k_{n} \in K$. Since $v(k)+n u(t) \neq 0$ holds for all $k \in K$, $n \in \mathbf{N}$, no $f \in K[t]$ satisfies $u(t f)=0$, i.e. $u$ is no valuation.

\section{REFERENCES}

1. N. Bourbaki, Elements of mathematics, commutative algebra, Hermann, Paris, AddisonWesley Publishing Co., Reading, Massachusetts, 1972

2. J. Gräter, Integral closure and valuation rings with zero-divisors, Studia Sci. Math. Hungarica 17 (1982), 457-458

3. J. A. Huckaba, Commutative rings with zero divisors, Monographs and Textbooks in Pure and Applied Mathematics, M. Dekker, Inc., New York and Basel, 1988

Institut für Algebra und Zahlentheorie, Technische Universität, PockelsStrasse 14, D-3300 BRAunschWeig, West Germany 